\title{
The dual continuity equations
}

\author{
A. I. Arbab (a) And Norah N. AlsaAwi (b) \\ ${ }^{a, b}$ Department of Physics, College of Science, Qassim University, P.O. Box 6644, Buraidah 51452, KSA \\ ${ }^{a}$ Department of Physics, Faculty of Science, University of Khartoum, P.O. Box 321, Khartoum 11115, Su- \\ dan
}

PACS

\begin{abstract}
The ordinary continuity equation relating the current and density of a system is extended to incorporate systems with dual (longitudinal and transverse) currents. Such a system of equations is found to have the same mathematical structure as that of Maxwell equations. The horizontal and transverse currents and the densities associated with them are found to be coupled to each other. Each of these quantities are found to obey a wave equation traveling at the velocity of light in vacuum. London's equations of super-conductivity are shown to emerge from some sort of continuity equations. The new London's equations are symmetric and are shown to be dual to each other. It is shown that London's equations are Maxwell's equations with massive electromagnetic field (photon). These equations preserve the gauge invariance that is broken in other massive electrodynamics. The duality invariance may allow magnetic monopoles to be present inside superconductors. The new duality is called the comprehensive duality transformation.
\end{abstract}

KEYWORDS: Continuity Equation; Maxwell Equations: Massive Electrodynamics; London's Superconductivity; Proca-Maxwell equations; Duality TRansformations

Introduction. - One of the most fundamental physical equation that any physical system should satisfy is the continuity equation. A closed electric system is known to comply with the continuity equation that manifests the charge conservation of the system. A current in physical world is generally an expression for the flow of a conserved quantity, like charge, baryon number, lepton number, etc. An electron, as by Schrodinger wave equation satisfies the continuity equation. Similarly, in the relativistic case, the electron is described by the Dirac equation, where a relativistic analogue of the continuity equation is shown to govern its motion [1]. At the same time, the propagation of sound in a medium is also described by a continuity equation. In fluid dynamics, the continuity equation expresses conservation of mass. Therefore, the continuity equation constitutes a fundamental integral equation in physical worlds. It also expresses the conservation of energy of a system.

In a relativistic world, the current density is expressed in terms of a 4-vector. This 4-vector is defined in terms of a temporal part in addition to a spatial vector part. The ordinary current vector density is represented by the spatial vector part, whereas the density is expressed by the fourth temporal part of the 4-vector. In

\footnotetext{
(a) arbab.ibrahim@gmail.com

(b) nooorah1994@gmail.com
} 


\section{A. I. Arbab ${ }^{1}$ and Norah N. Alsaawi ${ }^{2}$}

some representations, the scalar part is defined by an imaginary number, while the spatial part is a real number. To generalize this representation, we employ biquaternions, where the two numbers are generally complex [2]. Though the electromagnetic field is represented by a complex number, the current and charge densities were not.

We aim here to make this representation, and explore the physical significance of these complex quantities. We then derive the continuity equation that connects the temporal and spatial variations of current and charge densities. Recall that the electromagnetic wave arising from varying electric and magnetic fields, is known to propagate in a direction perpendicular to the plane spanned by the electric and magnetic fields. In ordinary systems a current flows along the horizontal direction but in others a possibility of a transverse is not ruled out. The continuity equation is known to be a single scalar equation. However, in the present formalism, a generalized set of continuity equations are obtained and their solution is found. These are shown to correspond to two scalar equations, and two vector equations. They have a common mathematical structure as that of the four Maxwell's equations. Remarkably, extending the continuity equation yields a symmetric form of London's equations of superconductivity [3]. Furthermore, the London's equations are found to emerge from Maxwell's equations when the electromagnetic field (photon) is allowed to be massive.

Quaternionic current density. - The continuity equation generally describes a conservation of charge, mass, quantum number, energy and momentum. It can be written in the general form [4]

$$
\vec{\nabla} \cdot \vec{J}+\frac{\partial \rho}{\partial t}=0
$$

where $\vec{J}$ and $\rho$ are the current and density of the system under consideration. For an electric system, for instance, $\vec{J}$ and $\rho$ define the electric current and charge densities. Inside a conductor whose electric conductivity is $\sigma$, the electric current and the electric field that causes the electron to flow follows the microscopic Ohm's law, $\vec{J}=\sigma \vec{E}$. This implies the current flows along the electric field direction only. One can also relate the current density to the flow of electric charges as $\vec{J}=\rho \vec{v}$. However, when a transverse magnetic field is applied to a conductor a transverse voltage is developed that is proportional to the electric field. Such an effect is found by Hall in 1885 .

The field equation of an inviscid fluid is described by Euler equation [4]. The field equation of a viscous fluid is described by the Navier-Stokes equation. This generalizes the Euler equation to include viscosity. A fluid flows when an external pressure is acted on it. The equation of motion of a viscous fluid is given by [4]

$$
\rho_{m} \frac{\partial \vec{v}}{\partial t}+\rho_{m} \vec{v} \cdot \vec{\nabla} \vec{v}=-\vec{\nabla} p+\eta \nabla^{2} \vec{v}
$$

where $\eta$ is the coefficient of viscosity, $p$ is the pressure, and $\rho_{m}$ is the mass density of the fluid. The coefficient $\eta$ could depend on external factors, e.g., temperature, density, etc. Other forces acting on the fluid can be added to the right hand-side of Eq.(2), e.g., gravity. A fluid can be characterised as compressible or incompressible (isotropic) depending on whether its density changes with space or not. An incompressible fluid is usually described by the equation, $\vec{\nabla} \cdot \vec{v}=0$. The solution of the Navier-Stokes equation is accompanied by the use of the continuity equation. At the same time, the solution of the Webster (Horn) equation that represents the propagation of sound in horns is also accompanied with the use of the continuity equation [5]. 
The dual continuity equations

To allow a current to flow along two normal directions, we represent it by a complex number, as $\vec{J}=\overrightarrow{J_{r}}+i \overrightarrow{J_{t}}$, or rather by a Biquaternion, and similarly the charge density by, $\rho=\rho_{r}+i \rho_{t}$.

For such a situation an extended continuity equation can be expressed as [2]

$$
\tilde{\nabla} \tilde{J}=0, \quad \tilde{J}=(i \rho, \vec{J}), \quad \tilde{\nabla}=\left(\frac{i}{c} \frac{\partial}{\partial t}, \vec{\nabla}\right), \quad \vec{J}=\overrightarrow{J_{r}}+i \vec{J}_{t}, \quad \rho=\rho_{r}+i \rho_{t} .
$$

Expanding Eq.(3) using the quaternionic product rule, and equating the real and imaginary parts for the scalar and vector parts of the resulting equations to zero, we obtain

$$
\vec{\nabla} \cdot \overrightarrow{J_{r}}+\frac{\partial \rho_{r}}{\partial t}=0, \quad \vec{\nabla} \cdot \overrightarrow{J_{t}}+\frac{\partial \rho_{t}}{\partial t}=0
$$

and

$$
\frac{\partial \overrightarrow{J_{r}}}{\partial t}+c^{2} \vec{\nabla} \rho_{r}+c \vec{\nabla} \times \overrightarrow{J_{t}}=0, \quad \frac{\partial \overrightarrow{J_{t}}}{\partial t}+c^{2} \vec{\nabla} \rho_{t}-c \vec{\nabla} \times \overrightarrow{J_{r}}=0 .
$$

Equations (3) and (4) represent the most general form that any system can have. Particular cases of Eqs.(3) and (4) can then be studied. We thus see from the above equations that the two currents and charges are coupled to each other in a similar way the electric and magnetic fields couple in Maxwell's equations. We already had an experience of the effect of the temporal variation of the counterpart of a give quantity described by some system of equations. Of such currents, is the displacement Maxwell's current. Such a system of equations will find applications in the field of hydrodynamics and plasma physics. The transverse current tends to lift the object (fluid) upward. In the Hall's effect when a magnetic field is applied on a sample, a transverse voltage occurs. In the quantum analogue, a horizontal resistivity vanishes when a transverse one occurs (at the plateau). Thus, two perpendicular currents can be assumed to be generated so that when one vanishes the second one is present.

London modelled the phenomenon of superconductivity as due to the flow of two currents; the ordinary electric current and a superelectric current that floats over it [3]. Thus, a question could arise if one can associate this super current to the transverse current above. Equations (3) and (4) are solved to give

$$
\frac{1}{c^{2}} \frac{\partial^{2} \rho_{r}}{\partial t^{2}}-\nabla^{2} \rho_{r}=0, \quad \frac{1}{c^{2}} \frac{\partial^{2} \rho_{t}}{\partial t^{2}}-\nabla^{2} \rho_{t}=0, \quad \frac{1}{c^{2}} \frac{\partial^{2} \overrightarrow{J_{r}}}{\partial t^{2}}-\nabla^{2} \overrightarrow{J_{r}}=0, \quad \frac{1}{c^{2}} \frac{\partial^{2} \overrightarrow{J_{t}}}{\partial t^{2}}-\nabla^{2} \overrightarrow{J_{t}}=0 .
$$

Hence, $\rho_{r}, \rho_{t}, \vec{J}_{r}, \vec{J}_{t}$, satisfy the wave equation travelling at the speed of light. It is thus interesting that any change in the charge of current densities in a place, will be immediately transmitted as a wave. However, using the ordinary continuity equation, one can't deduce that the current is a wave traveling at the speed of light.

The system of equations in Eqs.(4) and (5) reduces to our generalized continuity equation [2], when $\vec{J}_{t}=0$ and $\rho_{t}=0$. Equations (4) and (5) are dual to each others which are invariant under the duality transformation where $\overrightarrow{J_{r}} \rightarrow \overrightarrow{J_{t}}, \rho_{r} \rightarrow \rho_{t}$, and $\overrightarrow{J_{t}} \rightarrow-\vec{J}_{r}, \rho_{t} \rightarrow-\rho_{r}$. We therefore, call them the dual continuity equations. We encounter an analogous case in the Dirac formulation, where the spinor representation led to representing the Dirac particle with two spin-states [1], and moving with equal but opposite velocities. Once again, in Hall effect a transverse voltage (charge density) is developed without a current, when a transverse magnetic field is applied to a conductor/semiconductor. Such an effect can be accounted for upon employing Eqs.(4) and (5) by allowing $\overrightarrow{J_{t}}=0$, but $\rho_{t} \neq 0$. 
A. I. Arbab ${ }^{3}$ and Norah N. Alsaawi ${ }^{4}$

Now Eq.(5) can be manipulated to give

$$
\frac{1}{c} \frac{\partial I_{r}}{\partial t}=-\oint \overrightarrow{J_{t}} \cdot d \vec{\ell}
$$

and

$$
\frac{1}{c} \frac{\partial I_{t}}{\partial t}=\oint \overrightarrow{J_{r}} \cdot d \vec{\ell}
$$

using Stokes' and divergence theorems, where $I_{r}$ and $I_{t}$ are the current flowing along the horizontal and transverse directions. Note that the fluid circulation is given by $\Gamma=\oint \vec{v} \cdot d \vec{\ell}$, where for an ordinary fluid $\vec{J}=\rho \vec{v}$, and $\overrightarrow{J_{t}}=0$, and $\rho_{t}=0$ so that the current is steady, and that $\Gamma=0$. Note that the circulation around a closed contour $C$ is defined as the line integral of the velocity along that contour. One can now associate an electromotive force with the two current temporal variations, viz.,

$$
\frac{I_{r} R_{r}}{c L_{r}}=\frac{\epsilon_{r}}{c L_{r}}=\oint \vec{J}_{t} \cdot d \vec{\ell}, \quad \frac{I_{t} R_{t}}{c L_{t}}=\frac{\epsilon_{t}}{c L_{t}}=-\oint \overrightarrow{J_{r}} \cdot d \vec{\ell},
$$

where in electric system $\epsilon_{r}$ and $\epsilon_{t}$ are the electromotive forces pushing the $I_{r}$ and $I_{t}$ currents, $R_{r}$ and $R_{t}$ are the resistances of these currents, and $L_{r}$ and $L_{t}$ are the inductances due to $I_{r}$ and $I_{t}$ temporal variations, respectively. Equation suggests that two electric fields can be associated with $\vec{J}_{t}$ and $\vec{J}_{r}$ by

$$
\vec{E}_{r}=c L_{r} \vec{J}_{t}, \quad \vec{E}_{t}=-c L_{t} \vec{J}_{r}
$$

with their conductivities as $\sigma_{t}=1 /\left(L_{t} c\right)$ and $\sigma_{r}=1 /\left(L_{r} c\right)$. Hence, if we now express the velocity by $\vec{v}=\vec{v}_{r}+i \vec{v}_{t}$, then the total current can be related to the charge densities by the equation

$$
\vec{J}=\rho \vec{v}=\left(\rho_{r} v_{r}-\rho_{t} v_{t}\right)+i\left(\rho_{r} v_{t}+\rho_{t} v_{r}\right)=J_{r}+i J_{t}
$$

implying that

$$
J_{r}=\rho_{r} v_{r}-\rho_{t} v_{t}, \quad J_{t}=\rho_{r} v_{t}+\rho_{t} v_{r}
$$

It is interesting to see that $\vec{J}_{r} \neq \rho_{r} \vec{v}_{r}$ and $\vec{J}_{t} \neq \rho_{t} \vec{v}_{t}$.

Energy and momentum conservation equations. Now Eqs.(4) and (5) can be connected with an energy conservation equation that can be expressed as

$$
\frac{\partial u}{\partial t}+\vec{\nabla} \cdot \vec{S}=0
$$

where

$$
u=\frac{J_{t}^{2}}{2 c}+\frac{J_{r}^{2}}{2 c}+\frac{c \rho_{r}^{2}}{2}+\frac{c \rho_{t}^{2}}{2}, \quad \vec{S}=\overrightarrow{J_{t}} \times \overrightarrow{J_{r}}+c \rho_{r} \overrightarrow{J_{r}}+c \rho_{t} \overrightarrow{J_{t}}
$$

Note that Eq.(12) can also be seen as representing a continuity equation too. The momentum conservation equation can be obtained from Eqs.(4) and (5) where

$$
\frac{\partial g_{i}}{\partial t}+\partial_{j} T_{i j}-\vec{\nabla} \times\left(c^{2} \rho_{r} \overrightarrow{J_{t}}-c^{2} \rho_{t} \overrightarrow{J_{r}}\right)=0
$$


The dual continuity equations

where

$\vec{g}=\overrightarrow{J_{t}} \times \overrightarrow{J_{r}}-c \rho_{r} \overrightarrow{J_{r}}-c \rho_{t} \vec{J}_{t}, T_{i j}=\frac{c}{2}\left(J_{t}^{2}+J_{r}^{2}-c^{2} \rho_{r}^{2}-c^{2} \rho_{t}^{2}\right) \delta_{i j}-c\left(J_{r i} J_{r j}+J_{t i} J_{t j}\right)-\epsilon_{i j k} c^{2} \rho_{r} J_{r k}-\epsilon_{i j k} c^{2} \rho_{t} J_{t k}=0$.

Note that $T_{i j}$ can be see as a stress tensor, $u$ as an energy density, $\vec{S}$ as a Poynting vector, and $\vec{g}$ as a momentum density vector. In such conditions, the last term on the left hand-side of Eq.(13), will represent a force density acting on the fluid. It is a matter of interest if a fluid (with two components) satisfying the above equations really exists.

Current-charge densities transformation. It is very interesting to observe that Eqs.(4) and (5) are invariant under the charge-current densities transformation

$$
\vec{J}_{r}^{\prime}=\vec{J}_{t}+\vec{\nabla} f, \quad \rho_{r}{ }^{\prime}=\rho-\frac{1}{c^{2}} \frac{\partial f}{\partial t}
$$

and

$$
{\overrightarrow{J_{t}}}^{\prime}=\overrightarrow{J_{r}}+\vec{\nabla} g, \quad \rho_{t}{ }^{\prime}=\rho_{t}-\frac{1}{c^{2}} \frac{\partial g}{\partial t}
$$

where $f$ and $g$ are some scalar functions obeying a wave equation traveling at the speed of light, i.e.,

$$
\frac{1}{c^{2}} \frac{\partial^{2} f(g)}{\partial t^{2}}-\nabla^{2} f(g)=0
$$

Notice that the transformations of $\rho_{r}\left(\rho_{t}\right)$ and $\vec{J}_{r}\left(\vec{J}_{t}\right)$ are somehow analogous to the gauge transformations of the electromagnetic fields. Looking at Eqs.(4) and (5) with some scrutiny reveals that they have the same structure of the Maxwell's equations. The analogy dictates that while $\rho_{r}$ and $\rho_{t}$ are analogous to the electric and magnetic charges, the currents $\vec{J}_{r}$ and $\overrightarrow{J_{t}}$ are analogous to the electric and magnetic fields.

London's equations as continuity equations. - In his attempt to describe the superconductivity, London employed Newton's second law and Faraday equation [3]. He assumed that the superconductivity is transported by super-electrons. Super-electrons exist besides the normal electrons. He thus obtained the two equations $[3]$

$$
\frac{\partial \vec{J}}{\partial t}+c^{2} \vec{\nabla} \rho=\gamma \vec{E}, \quad \vec{\nabla} \times \vec{J}_{r}=-\gamma \vec{B}, \quad \gamma=\frac{n_{s} e^{2}}{m},
$$

where $n_{s}, e$ and $m$ are the number density of super-electrons, super-electronic charge and super-electron mass, respectively. We will show in the next section that these equations can be derived entirely from Maxwell's equations without resort to Newton's equation as done by London. They are in particular Maxwell's equations for a massive electromagnetic field.

Massive continuity equation. Since London's equations involve current and charge densities, one can treat them as a sort of continuity equation. We call this continuity the massive continuity equation for reasons to be disclosed later on. To see this, we express the continuity equation in Eq.(3) as $[7,8]$

$$
\tilde{\nabla} \tilde{J}=-\gamma \tilde{F}, \quad \tilde{F}=\left(\Lambda+i \Sigma, \vec{B}-\frac{i}{c} \vec{E}\right), \quad \tilde{A}=\left(\frac{i}{c} \varphi-\frac{1}{c} \psi, \vec{A}+i \vec{N}\right)
$$


A. I. Arbab ${ }^{5}$ and Norah N. Alsaawi ${ }^{6}$

where

$$
\Lambda=-\left(\vec{\nabla} \cdot \vec{A}+\frac{1}{c^{2}} \frac{\partial \varphi}{\partial t}\right), \quad \Sigma=-\left(\vec{\nabla} \cdot \vec{N}+\frac{1}{c^{2}} \frac{\partial \psi}{\partial t}\right)
$$

which can be seen as extending London's equations, Eq.(19), to the symmetric ones. Here $\vec{N}$ is the conjugate of $\vec{A}$ and $\psi$ is the conjugate of $\varphi$. In the standard electrodynamics, one has $\Lambda=0$ (Lorenz gauge condition) and that $\Sigma=0$.

Expanding Eq. (20) using the quaternionic product rule, one finds

$$
\vec{\nabla} \cdot \vec{J}_{r}+\frac{\partial \rho_{r}}{\partial t}=\gamma \Lambda, \quad \vec{\nabla} \cdot \vec{J}_{t}+\frac{\partial \rho_{t}}{\partial t}=\gamma \Sigma
$$

and

$$
\begin{array}{cc}
\frac{\partial \vec{J}_{r}}{\partial t}+c^{2} \vec{\nabla} \rho_{r}+c \vec{\nabla} \times \overrightarrow{J_{t}}=\gamma \vec{E}, & \vec{\nabla} \times \overrightarrow{J_{r}}-\frac{1}{c} \frac{\partial \overrightarrow{J_{t}}}{\partial t}-c \vec{\nabla} \rho_{t}=-\gamma \vec{B}, \\
\vec{E}=-\frac{\partial \vec{A}}{\partial t}-\vec{\nabla} \varphi+\vec{\nabla} \times \vec{K}, \quad \vec{B}=\vec{\nabla} \times \vec{A}+\frac{1}{c^{2}} \frac{\partial \vec{K}}{\partial t}+\vec{\nabla} W,
\end{array}
$$

where

$$
c \overrightarrow{J_{t}}=\gamma \vec{K}, \quad \vec{J}_{r}=-\gamma \vec{A}, \quad c^{2} \rho_{r}=-\gamma \varphi, \quad c \rho_{t}=\gamma W .
$$

The electric and magnetic fields definition in Eq.(24) are usually employed when studying magnetic monopoles in the framework of Maxwell's equations. Equation (25) shows that the dual vector and scalar potentials $(\vec{K}, W)$ are connected with the horizontal and transverse currents and charges densities, respectively. One of these connections had appeared in the London's theory of superconductivity. Equations (22) and (23) reduce to London's equations when $\Lambda=0, \Sigma=0, \rho_{t}=0$ and $\overrightarrow{J_{t}}=0$. It is interesting to see that the symmetric London's equations, Eqs.(22) and (23), are invariant under the duality transformations, where $\vec{E} \rightarrow c \vec{B}$ and $c \vec{B} \rightarrow-\vec{E}$ [6], then $\vec{J}_{r} \rightarrow \vec{J}_{t}, \vec{J}_{t} \rightarrow-\vec{J}_{r}$ and $\rho_{t} \rightarrow-\rho_{r}, \rho_{r} \rightarrow \rho_{t}$. Moreover, $\Lambda \rightarrow \Sigma$ and $\Sigma \rightarrow-\Lambda$. Therefore, the duality transformation is not restricted to the transformation of the electric and magnetic fields only [6]. This is so since Maxwell's equations connect the electromagnetic field to charge and current densities as well. We better call the above duality transformations as a comprehensive duality transformation. The comprehensive duality transformation is the same as that one follows from symmetric Maxwell's equations when magnetic monopoles are introduced.

A charge interaction with an electromagnetic field. If we now assume that the charges producing the currents $\vec{J}_{r}$ and $\vec{J}_{t}$ interact with the electromagnetic field, we use the momentum eigen value equation for the current density

$$
\tilde{\nabla} \tilde{J}^{\prime}=0, \quad \tilde{J}^{\prime}=\tilde{J}+\gamma \tilde{A}, \quad \tilde{J}=(i c \rho, \vec{J}), \quad \tilde{A}=\left(\frac{i}{c} \varphi, \vec{A}\right) .
$$

The above current replacement is tantamount to the minimal coupling, the momentum replacement for an interacting charge with an electromagnetic field. 
The dual continuity equations

Expanding Eq.(26) using the quaternionic product rule yields

$$
\vec{\nabla} \times \vec{J}+\gamma \vec{\nabla} \times \vec{A}=0, \quad \frac{1}{c} \frac{\partial \vec{J}}{\partial t}+\vec{\nabla} c \rho+\gamma\left(\frac{1}{c} \frac{\partial \vec{A}}{\partial t}+\frac{1}{c} \vec{\nabla} \varphi\right)=0, \quad \frac{\partial \rho}{\partial t}+\vec{\nabla} \cdot \vec{J}+\gamma\left(\frac{1}{c^{2}} \frac{\partial \varphi}{\partial t}+\vec{\nabla} \cdot \vec{A}\right)=0
$$

which reduces to

$$
\vec{\nabla} \times \vec{J}=-\gamma \vec{B}, \quad \frac{\partial \vec{J}}{\partial t}+c^{2} \vec{\nabla} \rho=\gamma \vec{E}, \quad \frac{\partial \rho}{\partial t}+\vec{\nabla} \cdot \vec{J}=0, \quad \frac{1}{c^{2}} \frac{\partial \varphi}{\partial t}+\vec{\nabla} \cdot \vec{A}=0,
$$

where

$$
\vec{E}=-\vec{\nabla} \varphi-\frac{\partial \vec{A}}{\partial t}, \quad \vec{B}=\vec{\nabla} \times \vec{A}
$$

It is interesting we derived the ordinary London's equations using the ordinary quaternionic representation of the $\tilde{A}$ and $\tilde{J}$ quaternions. Moreover, the charge conservation and the Lorenz gauge condition are associated with these London's equations. However, had we used the biquaternionic forms of $\tilde{A}$ and $\tilde{J}$, we would have obtained the symmetric (dual) London's equations with the charge conservation and the Lorenz gauge being relaxed.

Note that the full electrodynamics of superconductivity should be taken with Maxwell's equations. The Maxwell's equations in the symmetric form can be obtained via the biquaternionic equation $[7,8]$

$$
-\tilde{\nabla} * \tilde{F}=\mu_{0} \tilde{J}
$$

which upon using Eq.(3) and (20) yields

$$
\vec{\nabla} \cdot \vec{E}=\frac{\rho_{r}}{\varepsilon_{0}}+\frac{\partial \Lambda}{\partial t}, \quad \vec{\nabla} \cdot \vec{B}=c \mu_{0} \rho_{t}+\frac{1}{c} \frac{\partial \Sigma}{\partial t},
$$

and

$$
\vec{\nabla} \times \vec{B}-\frac{1}{c^{2}} \frac{\partial \vec{E}}{\partial t}=\mu_{0} \overrightarrow{J_{r}}-\vec{\nabla} \Lambda, \quad \vec{\nabla} \times \vec{E}+\frac{\partial \vec{B}}{\partial t}=-\mu_{0} c \vec{J}_{t}+c \vec{\nabla} \Sigma
$$

Interestingly, Eqs.(31) and (32) are invariant under the comprehensive duality transformations shown above. They are analogous to the symmetrized Maxwell's equations when the magnetic monopole is introduced. In this case $\rho_{t}$ and $\overrightarrow{J_{t}}$ are the corresponding magnetic charge and magnetic current densities, upon setting $\Lambda=0$ and $\Sigma=0$. Considering this situation, the London's superconductivity symmetric equations would allow a magnetic monopole and current to show up in superconductivity. It would then be a very interesting opportunity to welcome magnetic monopoles inside superconductors [9-11]. The London's superconductivity then describes a dual superconductivity. In such a system the electric field induces effects that are equivalent to those ones induced by the magnetic. Therefore, it is remarkable that working in symmetric electrodynamics would bring about many interesting electrodynamic phenomena.

Equations (31) and (32) yields

$$
\frac{1}{c^{2}} \frac{\partial^{2} \vec{E}}{\partial t^{2}}-\nabla^{2} \vec{E}=-\mu_{0}\left(\frac{\partial \overrightarrow{J_{r}}}{\partial t}+c^{2} \vec{\nabla} \rho_{r}+c \vec{\nabla} \times \overrightarrow{J_{t}}\right), \quad \frac{1}{c^{2}} \frac{\partial^{2} \vec{B}}{\partial t^{2}}-\nabla^{2} \vec{B}=\mu_{0}\left(\vec{\nabla} \times \overrightarrow{J_{r}}-\frac{1}{c} \frac{\partial \overrightarrow{J_{t}}}{\partial t}-c \vec{\nabla} \rho_{t}\right)
$$

and

$$
\frac{1}{c^{2}} \frac{\partial^{2} \Lambda}{\partial t^{2}}-\nabla^{2} \Lambda=-\mu_{0}\left(\vec{\nabla} \cdot \vec{J}_{r}+\frac{\partial \rho_{r}}{\partial t}\right), \quad \frac{1}{c^{2}} \frac{\partial^{2} \Sigma}{\partial t^{2}}-\nabla^{2} \Sigma=-\mu_{0}\left(\vec{\nabla} \cdot \overrightarrow{J_{t}}+\frac{\partial \rho_{t}}{\partial t}\right) .
$$




\section{A. I. $\operatorname{Arbab}^{7}$ and Norah N. Alsaawi ${ }^{8}$}

If we now assume Maxwell's equations, Eqs.(33) and (34), satisfy the Klein-Gordon equation of massive fields with mass $m_{f}$ then, Eqs.(33) and (34) reduce to

$$
\frac{1}{c^{2}} \frac{\partial^{2} \vec{E}}{\partial t^{2}}-\nabla^{2} \vec{E}=-\left(\frac{m_{f} c}{\hbar}\right)^{2} \vec{E}, \quad \frac{1}{c^{2}} \frac{\partial^{2} \vec{B}}{\partial t^{2}}-\nabla^{2} \vec{B}=-\left(\frac{m_{f} c}{\hbar}\right)^{2} \vec{B}
$$

and

$$
\frac{1}{c^{2}} \frac{\partial^{2} \Lambda}{\partial t^{2}}-\nabla^{2} \Lambda=-\left(\frac{m_{f} c}{\hbar}\right)^{2} \Lambda, \quad \frac{1}{c^{2}} \frac{\partial^{2} \Sigma}{\partial t^{2}}-\nabla^{2} \Sigma=-\left(\frac{m_{f} c}{\hbar}\right)^{2} \Sigma
$$

where

$$
\begin{array}{rr}
\left(\frac{m_{f} c}{\hbar}\right)^{2} \vec{E}=\mu_{0}\left(\frac{\partial \vec{J}_{r}}{\partial t}+c^{2} \vec{\nabla} \rho_{r}+c \vec{\nabla} \times \overrightarrow{J_{t}}\right), \quad\left(\frac{m_{f} c}{\hbar}\right)^{2} \vec{B}=-\mu_{0}\left(\vec{\nabla} \times \vec{J}_{r}-\frac{1}{c} \frac{\partial \overrightarrow{J_{t}}}{\partial t}-c \vec{\nabla} \rho_{t}\right), \\
\left(\frac{m_{f} c}{\hbar}\right)^{2} \Lambda=\mu_{0}\left(\vec{\nabla} \cdot \vec{J}_{r}+\frac{\partial \rho_{r}}{\partial t}\right), \quad\left(\frac{m_{f} c}{\hbar}\right)^{2} \Sigma=\mu_{0}\left(\vec{\nabla} \cdot \vec{J}_{t}+\frac{\partial \rho_{t}}{\partial t}\right) .
\end{array}
$$

These equations are but the dual London's equations of superconductivity, Eq.(23), if we let

$$
\left(\frac{m_{f} c}{\hbar}\right)^{2}=\mu_{0} \gamma
$$

Therefore, the London's equations of superconductivity implicitly imply the electromagnetic field (photon) is massive. Note that $\gamma$ is a measure of the photon mass, and this is why we call the continuity equation, Eq.(20), the massive continuity equation. It is remarkable that the present massive electrodynamics doesn't break the gauge invariance. Thus, the photon acquires a mass without violating the gauge invariance that is normally broken in other massive electrodynamics theories. It is interesting to see that Eq.(33) and Eq.(34) are dual. One remarks that the London's equations of superconductivity are rather generic equations of the electromagnetic field, and is not particularly linked to superconductors.

Now integrating the second equation in Eq.(23), with respect to the area element, and using the Stokes and divergence theorems, one finds

$$
\frac{\partial I_{r}}{\partial t}+c \oint \overrightarrow{J_{t}} \cdot d \vec{\ell}=\gamma \phi_{E}, \quad \frac{1}{c} \frac{\partial I_{t}}{\partial t}-\oint \overrightarrow{J_{r}} \cdot d \vec{\ell}=\gamma \phi_{B}
$$

where $\phi_{E}$ and $\phi_{B}$ are the electric and magnetic fluxes enclosed by the surface, respectively. It is thus apparent that a magnetic field arises from the circulation of $\vec{J}_{r}$ and the temporal variation of $I_{t}$. Because of the presence of the factor $c$ in the numerator of the latter term, we expect its contribution in ordinary conditions to be very small. Equation (41) shows that the electric and magnetic fluxes in the dual London's paradigm are different from that in the normal electrodynamics. A time varying of the transverse current reduces the magnetic flux, and the circulation of a horizontal current increases the electric flux.

The dual Lorentz force. Owing to the above formulation, one can define the dual Lorentz force as

$$
\tilde{f}=q \tilde{V} \tilde{F}, \quad \tilde{V}=\left(i c, \vec{v}_{r}+i \vec{v}_{t}\right),
$$

which can be expressed in the form

$$
\tilde{f}=\left(\frac{i}{c}\left(P_{r}+i P_{t}\right), \overrightarrow{f_{r}}+i \overrightarrow{f_{t}}\right),
$$


The dual continuity equations

and upon using the quaternionic product rule, reduces to

$$
P_{r}=q c^{2} \Lambda+q \vec{v}_{r} \cdot E-q c \vec{v}_{t} \cdot \vec{B}, \quad P_{t}=q c^{2} \Sigma+q c \vec{v}_{r} \cdot \vec{B}+q \vec{v}_{t} \cdot \vec{E},
$$

and

$$
\overrightarrow{f_{r}}=\left(q \vec{E}+q \vec{v}_{r} \times \vec{B}+q \Lambda \vec{v}_{r}\right)-q \vec{v}_{t} \Sigma+\frac{q}{c} \vec{v}_{t} \times \vec{E}, \quad \overrightarrow{f_{t}}=q c \vec{B}-\frac{q}{c} \vec{v}_{r} \times \vec{E}+q \vec{v}_{r} \Sigma+\left(q \Lambda \vec{v}_{t}+q \vec{v}_{t} \times \vec{B}\right)
$$

The above equations can be reduced to any particular case we wish to study. We thus have formulated all physical equations describing the dynamics of fluids governed by two perpendicular currents flow. The two forces in Eq.(43) are dual to each other, and the two powers in Eq.(44) are dual to each other too. It is very interesting that under the duality transformation, $\vec{v}_{r} \rightarrow \vec{v}_{r}$ and $\vec{v}_{t} \rightarrow \vec{v}_{t}$, that is consistent with Eq.(11). It is interesting to see that there is an additional force appearing in $\vec{f}_{r}$ on a moving charge if the theory admits a violation of the Lorenz gauge condition $(\Lambda \neq 0)$. This drag force is present in the absence of electric and magnetic field. Since it depends on the velocity of the particle, it can be connected with a presence of a fluid (aether) in which the charge floats. The scalar $\Lambda$ can be connected with some remnant magnetic scalar field exists in space-time.

Extended Maxwell - Proca equations. - Maxwell-Proca equations generalize Maxwell's equations to include massive electromagnetic field [12]. To derive these equations, we employ the current density definition in Eq.(26) and apply it in Eq.(30) to obtain

$$
-\tilde{\nabla}^{*} \tilde{F}=\mu_{0}(\tilde{J}-\gamma \tilde{A}), \quad \tilde{F}=\left(\Lambda, \vec{B}-\frac{i}{c} \vec{E}\right),
$$

which upon using the quaternionic product rule, one finds

$$
\vec{\nabla} \cdot \vec{E}=\frac{\rho}{\varepsilon_{o}}-\mu_{0} \gamma \varphi, \quad \vec{\nabla} \cdot \vec{B}=\frac{1}{c} \frac{\partial \Lambda}{\partial t},
$$

and

$$
\vec{\nabla} \times \vec{E}=-\frac{\partial \vec{B}}{\partial t}+c \vec{\nabla} \Lambda, \quad \vec{\nabla} \times \vec{B}=\frac{1}{c^{2}} \frac{\partial \vec{E}}{\partial t}+\mu_{0} \vec{J}-\mu_{0} \gamma \vec{A}
$$

These are the Maxwell - Proca equations if we use Eq.(39) and set $\Lambda=0$. Therefore, Eqs.(46) and (47) generalize the Maxwell - Proca equations. Note that the original Maxwell - Proca equations assume that the Lorenz gauge condition is valid. It is interesting that Eqs.(46) and (47) express massive electrodynamics with magnetic charge and current densities arising from the temporal and spatial variations of $\Lambda$. Here, $\Lambda$ satisfies a wave equation traveling at the speed of light in vacuum.

The energy equation connected with Eq.(46) and (47) is

$$
\vec{\nabla} \cdot \vec{S}_{\Lambda}+\frac{\partial u_{\Lambda}}{\partial t}=-\vec{E} \cdot \vec{J}-\gamma \Lambda \varphi
$$

where

$$
\vec{S}_{\Lambda}=\frac{\vec{E} \times \vec{B}-c \Lambda \vec{B}}{\mu_{0}}+\gamma \varphi \vec{A}, \quad u_{\Lambda}=\frac{1}{2} \varepsilon_{0} E^{2}+\frac{B^{2}}{2}+\frac{\Lambda^{2}}{2}+\frac{\gamma}{2 c^{2}} \varphi^{2}+\frac{\gamma}{2} A^{2}
$$


A. I. $\operatorname{Arbab}^{9}$ and Norah N. Alsaawi 10

Equation (48) shows that $\Lambda$ is coupled to $\varphi$. Note that in vacuum $\rho=0, \vec{J}=0$ and $\Lambda=0$.

The wave equations associated with Eqs.(46) and (47) can be found upon using the quaternionic equation

$$
-\tilde{\nabla}\left(\tilde{\nabla}^{*} \tilde{F}\right)=\mu_{0} \tilde{\nabla}(\tilde{J}-\gamma \tilde{A}) .
$$

Concluding remarks. - We have studied a symmetric system of continuity equations that generalises the ordinary one. A particular case of continuity equations is found that yields a symmetric form of the London's equations of superconductivity. These new equations are found to be invariant under duality transformation. Symmetric Maxwell's equations, that generalize the commonly known ones where the Lorenz gauge condition is not respected, are derived. A massive electrodynamics preserving the gauge invariance is obtained. London's equations are shown to represent Maxwell's equations when an electromagnetic field is allowed to be massive. Maxwell - Proca equations are generalized to the case where the Lorenz gauge condition is relaxed. It is thus apparent that it is very convenient to use quaternions to formulate our physical laws.

\section{REFERENCES}

[1] Bjorken, J. D., and DRell, S. D., Relativistic Quantum Fields, McGraw-Hill College, (1965).

[2] Arbab, A. I., and Widatallah, H. M., The geenralized continuity equation, Chinese Phys. Lett. 27, 084703 (2010).

[3] London, F., and London, H., The Electromagnetic Equations of the Supraconductor, Proc. Roy. Soc. A149, 71 (1935).

[4] Landau, L. D., and Lifshitz, E. M., Fluid mechanics, Course of Theoretical Physics, Pergamon Press, (1987).

[5] Webster, A. G., Acoustical impedance, and the theory of horns and of the phonograph, Proc. Natl. Acad. Sci. USA, 5 (1919).

[6] Heaviside, G. , Some properties of Maxwell equations, Phil. Trans. Roy. Soc.A 183, 423 (1893).

[7] Arbab, A. I., Quantized Maxwell's equations, Optik, 136, 64 (2017).

[8] Arbab, A. I., Extended electrodynamics and its consequences, Mod. Phys. Lett. B, 31, 1750099 (2017).

[9] Cabrera, B., First Results from a Superconductive Detector for Moving Magnetic Monopoles, Phys. Rev. Lett., Vol.48, (1982).

[10] Taylor, J. D. , Ross, R. R., Eberhard, P. H., and Byrns, R. A., Superconducting Elements of a Magnetic Monopole Detector, Journal of Applied Physics 42, 5 (1971).

[11] Tassie, L.J., Dirac magnetic monopoles and superconductivity, Nuovo Cim. 38, 1935 (1965).

[12] Proca, A., J. Phys. Radium 7347 (1936).

[13] Jackson, J. D., Classical Electrodynamics, John Wiley \& Sons, New York, (1998). 